\title{
Factors Associated with Recovered Functionality After Hip Fracture in Non-Institutionalized Older Adults: A Case-Control Study Nested in a Cohort
}

\author{
Eliseo Ramírez-García (D) \\ Guadalupe S García de la \\ Torre $\mathbb{D}^{2}$ \\ Erika Judith Rodríguez Reyes ${ }^{3}$ \\ Karla Moreno-Tamayo (D) ${ }^{1}$ \\ María Claudia \\ Espinel-Bermudez $\mathbb{D}^{4}$ \\ Sergio Sánchez-García (iD) \\ 'Epidemiological and Health Services \\ Research Unit, Aging Area, National \\ Medical Center XXI Century, Instituto \\ Mexicano del Seguro Social, Mexico City, \\ Mexico; ${ }^{2}$ Public Health Department, \\ School of Medicine, Universidad Nacional \\ Autónoma de México, Mexico City, \\ Mexico; ${ }^{3}$ Epidemiological Surveillance \\ Coordination, Instituto Mexicano del \\ Seguro Social, Mexico City, Mexico; \\ ${ }^{4}$ Clinical Epidemiology Research Unit, \\ UMAE Specialties Hospital, Western \\ Medical Center, Instituto Mexicano del \\ Seguro Social, Guadalajara, México
}

\begin{abstract}
Purpose: To identify factors associated with recovered functionality after a hip fracture in a sample of older adult patients.

Patients and Methods: Nested case-control study in a cohort. Older adults (60 years or older) with a hip fracture were recruited between May 2017 and October 2018. The Barthel scale was used to measure performance in activities of daily living (ADL). A questionnaire was applied to collect information about demographic, clinic, psychological and social variables, and anthropometric measurements were taken. A logistic regression model was built to analyze various factors related to recovered functionality.
\end{abstract}

Results: A total of 346 older adults with a hip fracture were studied ( $\mathrm{n}=173$ cases and $\mathrm{n}=173$ controls); $69.4 \%(\mathrm{n}=240)$ women and $30.6 \%(\mathrm{n}=140)$ men. Mean age was 79.4 years $( \pm 8.7)$ overall; for cases, $77.4( \pm 7.9)$ years and for controls, $81.4( \pm 9.0)$. Mean schooling was $6.3( \pm$ 4.3) years. Recovered functionality was associated with normal nutritional status (OR 4.81, $95 \% \mathrm{CI}=2.54-9.12)$, absence of heart disease (OR 4.08, 95\% CI $=1.48-11.20)$, selfefficacy for ADL (OR 4.07, 95\% CI $=2.15-7.72)$, absence of depressive symptoms (OR $2.99,95 \% \mathrm{CI}=1.69-5.28)$, prior functionality $(\mathrm{OR} 2.83,95 \% \mathrm{CI}=1.51-5.31)$, high socioeconomic level (OR 2.41, 95\% CI $=1.24-4.65)$ and transcervical fracture (OR 2.34, $95 \% \mathrm{CI}=1.05-5.22$ ).

Conclusion: In older adults who have suffered a hip fracture, clinical, psychological, and demographic characteristics are associated with recovered functionality. These factors should be considered as a priority in the care of older adults who have experienced hip fractures.

Keywords: functionality, hip fracture, older adults, case-control studies

\section{Introduction}

Hip fractures have become an important public health problem due to their increased frequency ${ }^{1}$ and high impact on older adults' functionality, quality of life and mortality. ${ }^{2}$ A large number of older adults do not regain functionality despite receiving timely medical care and rehabilitation, suggesting that nonclinical factors also influence recovery. ${ }^{3}$

In several studies, advanced age, ${ }^{4}$ the type of fracture, ${ }^{5}$ hip pain, nutritional status, ${ }^{7}$ comorbidities, ${ }^{8}$ and functionality prior to the fracture ${ }^{9}$ have played a part in functional recovery at different moments after discharge. Others have evaluated factors related to the psychosocial context, such as social network, occupational level, emotional state and cognitive state. ${ }^{6,10,11}$ However, few studies have exhaustively explored the influence of social characteristics on recovery of hip fracture in
Correspondence: Sergio Sánchez-García Email sergio.sanchezga@imss.gob.mx 
older adults, such as socioeconomic status or experiencing loneliness. Likewise, psychological traits including selfefficacy in activities of daily living (ADL), perceived health, or fear of falling have not been appropriately studied with medical conditions. On the other hand, results from the few existing studies have not been supported by measurements made with accurate and valid instruments; in most, predictors stem from a functional loss perspective, not considering recovery as a successful result. Identifying elements that favor a return to health and functional recovery are very useful for developing appropriate and efficient prevention, intervention and rehabilitation programs. ${ }^{8}$ Due to these reasons, we conducted this study with the objective of identifying factors associated with recovered functionality after a hip fracture in a sample of older adult patients, considering demographic, clinical, social and psychological characteristics.

\section{Materials and Methods Design and Sample}

This was a case-control study nested in a dynamic prospective cohort that aimed to evaluate functionality and mortality in Mexican older adults with a hip fracture. The cohort study included 505 participants, beneficiaries of the Instituto Mexicano del Seguro Social (IMSS) in Mexico City, who lived in the community and had a hip fracture. The participants were recruited by consecutive sampling from May 2017 to October 2018 at the Regional General Hospital number 2 (HGR2) "Dr Guillermo Fajardo Ortiz", which provides care to beneficiaries who live in a geographic area corresponding to 14 of the 16 municipalities in Mexico City. The follow-up period consisted of the moment of hospitalization for hip fracture to 12 months later; during this period, 4 follow-up measurements were scheduled (1, 3, 6 and 12 months). All patients admitted to the trauma and orthopedic unit with a hip fracture diagnosis were invited to participate and required to sign a consent form. The inclusion criteria for the cohort were as follows: Hospital admission for hip fracture generated by low-impact trauma such as falling when standing or walking and age 60 or older. Patients who had an amputation or absence of any limb, blindness or deafness, hip fracture associated with a tumor, metastasis or high-impact multiple trauma were excluded.

For the case-control study nested in a cohort, a minimum sample size was estimated according to the Schlesselman formula for case-control studies, ${ }^{12}$ in which the absence of depression (83.5\%) associated with functionality after hip fracture (OR 6.37) ${ }^{13}$ was considered an exposure factor for recovery (power of $80 \%$ and an alpha value of $5 \%$ ). The minimum sample size was 138 individuals; however, for the purposes of this study, all available cases were included, so the sample was made up of 173 cases (functional) and 173 controls (non-functional). From the cohort subjects, those whose hip fracture had occurred more than 3 months prior to the study and who did not have a medical diagnosis of dementia were selected (Figure 1). The patients were located via telephone to set up an appointment for an interview to obtain general, demographic, clinical, social and psychological data. Evaluations were performed by qualified health personnel.

\section{Case-Control Identification}

\section{Functionality}

Functionality in the basic activities of daily living (ADL) before and after the hip fracture was measured using Barthel's index. The previous functionality was obtained retrospectively (last month before the hip fracture) and the current one at the time of the interview. A score from 0 to 100 was obtained, where a higher score indicated greater functionality. ${ }^{14}$ Functional cases were identified with a score $>90$ points and controls (randomly selected) with a score $\leq 90$ points. ${ }^{15}$

\section{Exposure Variables Demographic Variables}

These included sex (woman/man); age in years (60-69/70$79 / 80-89 / \geq 90$ ); marital status (partner/no partner); socioeconomic status (high/medium and low), measured with a previously validated questionnaire in the Mexican population; ${ }^{16}$ and employment status, which was dichotomized as not employed or unemployed according to status at the time of the interview.

\section{Clinical Variables}

Comorbidities such as diabetes mellitus type 2 (DM2), systemic arterial hypertension ( $\mathrm{SAH}$ ), osteoporosis, history of depression, osteoarthritis, cerebrovascular disease (CVD), hemiplegia, chronic kidney disease, chronic respiratory diseases, and heart disease (myocardial infarction, heart failure, arrhythmia and heart valve disease) were investigated by direct questioning and corroborated with clinical records. In addition, general comorbidity was measured using the Charlson index and classified into two categories (Low: $<3$ points/High: $\geq 3$ points). The type of fracture according to anatomical position (sub capital/ 


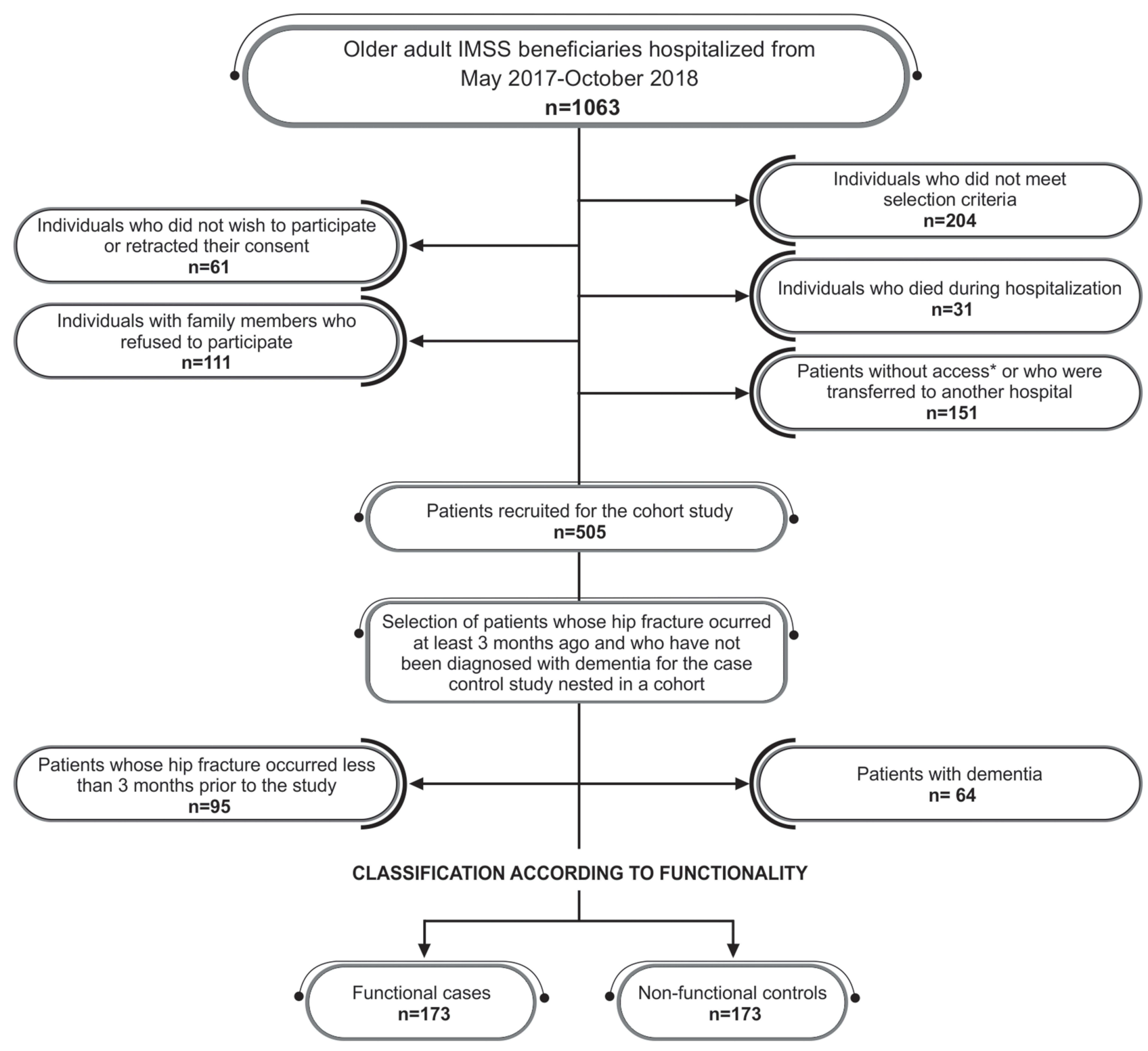

Figure I Study flow chart of the case control study nested in a cohort of subjects with and without functionality.

Notes: *Patients who encountered difficulties for various reasons: clinical, paraclinical, or preoperative evaluations that interfered with measurements during hospitalization.

Abbreviation: IMSS, Mexican Institute of Social Security.

transcervical/basicervical/transtrochanteric/subtrochanteric) was obtained from the clinical record. The Mini Nutritional Assessment (MNA) was used to identify normal nutritional status ( $>24$ points), nutritional risk, and malnutrition ( $\leq 24$ points). The intensity of self-perceived hip pain was obtained by direct questioning based on a numerical scale from 0 to 10 and it was categorized as follows: high (8-10 points), moderate (4-7 points), low (1-3 points) and no pain ( 0 points). ${ }^{17}$ The length of rehabilitation was measured in number of months and two categories were constructed ( $\geq 1 \mathrm{month} /<1$ month).

\section{Social Variables}

Social support was measured with the Scale of Social Support Networks for Older Adults (ERASAM, for its Spanish acronym), designed and validated for Mexican older adults. It has a global score that ranges from 0 to 100; a higher score indicates a high support network. From the score, 3 categories were generated according to tertiles of the sample (high ( $\geq 57$ points)/moderate (44-56 points)/ low ( $\leq 43$ points)). ${ }^{18}$ The experience of loneliness was measured with the loneliness scale for elders, validated in the Mexican population, ${ }^{19}$ which is classified into 4 
categories (never $(<20$ points)/ almost never (20-39 points)/ sometimes (40-59 points)/ most of the time $(\geq 60$ points)).

\section{Psychological Variables}

The presence of clinically significant depression symptoms was identified with the Center for Epidemiologic Studies' Depression Scale (Revised) (CESD-R). ${ }^{20}$ Depression was determined with a cut-off of $\leq 57$ points. $^{20}$ The MiniMental State Examination (MMSE) was used to measure cognitive impairment considering a cut-off point of $\leq 23$, adjusted for schooling. ${ }^{21}$ Self-efficacy to perform daily activities was also measured at two levels (low and moderate ( $<57$ points)/high ( $\geq 57$ points)), using a previously validated questionnaire in the Mexican population. ${ }^{22}$ Selfperception of health was obtained with the Beaman scale. The total score was categorized into very good $(\geq 45$ points), good (42-44 points), poor (38-41 points), and very poor ( $\leq 37$ points) based on the quartiles of the entire sample. $^{23}$ Fear of falling was measured with the Falls Efficacy Scale-International (FES-I) ${ }^{24}$ in three categories: (low ( $\leq 44$ points)/moderate $(45-55$ points)/high $(\geq 56$ points)), constructed from the tertiles of the sample.

\section{Statistical Analysis}

Descriptive analysis included absolute and relative frequencies. Differences between cases (functional) and controls (non-functional) were identified with Chi-square tests $\left(\mathrm{X}^{2}\right)$ or Fisher's exact test. The bivariate and multivariable logistic regression analysis were used to determine the strength of association (Odds Ratio, OR; 95\% Confidence Interval, 95\% CI) between functionality and demographic, clinical, social and psychological characteristics. From the crude OR, variables with $\mathrm{p} \leq 0.20$ were identified. Thus, a multiple logistic regression analysis with backward elimination was performed; during this process variables with values close to $p=1.0$ were identified and removed one by one until the model reached statistical significance $(\mathrm{p} \leq 0.05)$. The final model was built with only significant variables to explain functionality after hip fracture (adjusted OR). To rule out collinearity, the variance inflation factor $(\mathrm{VIF}<10)$ was estimated. The model was adjusted using the Wald test; The Hosmer and Lemeshow test was used for its validation. Statistical analysis was performed with STATA version 14 software (StataCorp 2015).

\section{Results}

Three hundred and forty-six older adults with a hip fracture ( $\mathrm{n}=173$ cases and $\mathrm{n}=173$ controls) were studied; $69.4 \%(n=240)$ women and $30.6 \%(n=140)$ men. The mean age was 79.4 years $( \pm 8.7)$; for the cases it was 77.4 $( \pm 7.9)$ and for the controls it was $81.4( \pm 9.0)$. Mean level of schooling was $6.3( \pm 4.3)$ years. The most frequent chronic diseases were: SAH (60.4\%), DM2 (36.1\%), osteoporosis $(17.9 \%)$, osteoarthritis $(14.5 \%)$, and $59.8 \%$ of the patients had a transtrochanteric fracture.

The frequencies of the characteristics were studied, as well as the strength of association (crude OR, 95\% CI) with functionality after hip fracture; these are presented in Table 1. Statistically significant strength of association between both groups indicated that age $>90$ years, cerebrovascular disease, hemiplegia, heart disease, high comorbidity, transtrochanteric fracture, severe hip pain, presence of current depressive symptoms and cognitive impairment were more frequent in non-functional patients. On the other hand, high socioeconomic level, being active at work, presence of DM2, being functional prior to the fracture, transcervical fracture, normal nutritional status, receiving rehabilitation $>1$ month, high social support network, experiencing low levels of loneliness, high levels of self-efficacy for activities of daily living, good self-perception of health, and low fear of falling were characteristics that predominated in patients who were functional after a hip fracture.

Table 2 shows the final multiple logistic regression model and the strength of association (adjusted OR, 95\% $\mathrm{CI})$. The model indicates that the variables associated with recovered functionality after hip fracture are: Normal nutritional status $(4.81,2.54-9.12)$, absence of heart disease (4.08, 1.48-11.20), having a high level of self-efficacy for activities of daily living $(4.07,2.15-7.72)$, no current depressive symptoms $(2.99,1.69-5.28)$, being functional prior to the fracture $(2.83,1.51-5.31)$, high socioeconomic status $(2.41,1.24-4.65)$ and transcervical fracture $(2.34$, 1.05-5.22). No multicollinearity was observed between the variables (correlation matrix $<60 \%$ ). The variance inflation factor (VIF) corroborated the absence of collinearity (the predictor of all VIF $<10$ ). The Hosmer and Lemeshow test showed a good fit $(\mathrm{p}=0.868)$.

\section{Discussion}

We found that normal nutritional status, absence of heart disease, high level of self-efficacy for activities of daily living, absence of current depressive symptoms, 
Table I Characteristics Studied and Strength of Association (Crude OR, 95\% Cl) with Functionality After Hip Fracture

\begin{tabular}{|c|c|c|c|c|c|c|c|c|c|c|}
\hline \multirow[t]{2}{*}{ Variable } & \multicolumn{2}{|c|}{$\begin{array}{c}\text { Total } \\
n=346\end{array}$} & \multicolumn{2}{|c|}{$\begin{array}{c}\text { Cases } \\
\text { Functional } \\
n=173\end{array}$} & \multicolumn{2}{|c|}{$\begin{array}{l}\text { Controls Non } \\
\text { Functional } \\
n=173\end{array}$} & \multirow[t]{2}{*}{$p$ - value } & \multirow[t]{2}{*}{ ORc } & \multirow[t]{2}{*}{$95 \% \mathrm{Cl}$} & \multirow[t]{2}{*}{ p - value } \\
\hline & $\mathbf{n}$ & (\%) & $\mathbf{n}$ & (\%) & $\mathbf{n}$ & (\%) & & & & \\
\hline \multicolumn{11}{|l|}{ Sex } \\
\hline Men & 106 & $(30.6)$ & 56 & $(32.4)$ & 50 & $(28.9)$ & 0.41 & 1.17 & $0.74-1.86 \mid$ & 0.48 \\
\hline Women & 240 & $(69.4)$ & 117 & $(67.6)$ & 123 & (7I.I) & 0.58 & 1 & - & - \\
\hline \multicolumn{11}{|l|}{ Age (Years) } \\
\hline $60-69$ & 49 & $(14.2)$ & 29 & $(16.8)$ & 20 & $(11.6)$ & 0.06 & 4.23 & $1.81-9.90$ & 0.001 \\
\hline $70-79$ & 126 & $(36.4)$ & 69 & (39.9) & 57 & (32.9) & 0.13 & 3.53 & I.72-7.27 & 0.001 \\
\hline $80-89$ & 120 & $(34.7)$ & 62 & $(35.8)$ & 58 & $(33.5)$ & 0.60 & 3.12 & I.5I-6.44 & 0.002 \\
\hline$\geq 90$ & 51 & $(14.7)$ & 13 & $(7.5)$ & 38 & $(22.0)$ & $<0.001$ & 1 & - & - \\
\hline \multicolumn{11}{|l|}{ Marital status } \\
\hline Has partner & 129 & (37.3) & 64 & $(37.0)$ & 65 & $(37.6)$ & 0.90 & 0.97 & $0.63-1.508$ & 0.91 \\
\hline No partner & 217 & $(62.7)$ & 109 & $(63.0)$ & 108 & $(62.4)$ & 0.92 & I & - & - \\
\hline \multicolumn{11}{|l|}{ Socioeconomic status } \\
\hline High & 259 & $(74.9)$ & 145 & $(83.8)$ & 114 & $(65.9)$ & 0.006 & 2.68 & $1.60-4.47$ & $<0.001$ \\
\hline Medium and low & 68 & $(19.7)$ & 28 & $(11.6)$ & 59 & $(27.7)$ & $<0.001$ & I & - & - \\
\hline \multicolumn{11}{|l|}{ Employment status } \\
\hline Employed & 71 & $(20.5)$ & 49 & $(28.3)$ & 22 & $(12.7)$ & $<0.001$ & 2.71 & $1.55-4.73$ & $<0.001$ \\
\hline Unemployed & 275 & $(79.5)$ & 124 & (7I.7) & $|5|$ & $(87.3)$ & 0.021 & 1 & - & - \\
\hline \multicolumn{11}{|l|}{ Diabetes Mellitus type 2} \\
\hline Absent & 221 & $(63.9)$ & 102 & $(59.0)$ & 119 & $(68.8)$ & $<0.001$ & 0.65 & $0.4 I-1.0 \mathrm{I}$ & 0.05 \\
\hline Present & 125 & $(36.1)$ & 71 & $(4 I .0)$ & 54 & $(31.2)$ & 0.032 & I & - & - \\
\hline \multicolumn{11}{|l|}{ Systemic arterial hypertension } \\
\hline Absent & 137 & $(39.6)$ & 71 & $(4 I .0)$ & 66 & $(38.2)$ & 0.54 & 1.12 & $0.73-1.737$ & 0.58 \\
\hline Present & 209 & $(60.4)$ & 102 & $(59.0)$ & 107 & $(61.8)$ & 0.62 & 1 & - & - \\
\hline \multicolumn{11}{|l|}{ Osteoporosis } \\
\hline Absent & 284 & $(82.1)$ & 138 & $(79.8)$ & 146 & $(84.4)$ & 0.50 & 0.72 & $0.4 I-1.268$ & 0.26 \\
\hline Present & 62 & $(17.9)$ & 35 & $(20.2)$ & 27 & $(15.6)$ & 0.15 & 1 & - & - \\
\hline \multicolumn{11}{|l|}{ History of Depression } \\
\hline Absent & 325 & (93.9) & 165 & $(95.4)$ & 160 & $(92.5)$ & 0.69 & 1.67 & $0.67-4.15 \mid$ & 0.26 \\
\hline Present & 21 & $(6.1)$ & 8 & $(4.6)$ & 13 & $(7.5)$ & 0.12 & I & - & - \\
\hline \multicolumn{11}{|l|}{ Osteoarthritis } \\
\hline Absent & 296 & $(85.5)$ & 150 & $(86.7)$ & 146 & $(84.4)$ & 0.72 & 1.20 & $0.66-2.200$ & 0.54 \\
\hline Present & 50 & $(14.5)$ & 23 & $(13.3)$ & 27 & $(15.6)$ & 0.42 & I & - & - \\
\hline \multicolumn{11}{|l|}{ Cerebral Vascular Disease } \\
\hline Absent & 329 & $(95.1)$ & 170 & $(98.3)$ & 159 & (91.9) & 0.39 & 4.99 & $1.40-17.68$ & 0.013 \\
\hline Present & 17 & $(4.9)$ & 3 & $(1.7)$ & 14 & $(8.1)$ & $<0.001$ & 1 & - & - \\
\hline \multicolumn{11}{|l|}{ Hemiplegia } \\
\hline Absent & 336 & $(97.1)$ & $17 \mid$ & $(98.8)$ & 165 & $(95.4)$ & 0.64 & 4.14 & $0.86-19.81$ & 0.07 \\
\hline Present & 10 & $(2.9)$ & 2 & $(1.2)$ & 8 & $(4.6)$ & 0.007 & I & - & - \\
\hline \multicolumn{11}{|l|}{ Heart disease } \\
\hline Absent & 312 & $(90.2)$ & 165 & $(95.4)$ & 147 & $(85.0)$ & 0.15 & 3.64 & $1.60-8.30$ & 0.002 \\
\hline Present & 34 & $(9.8)$ & 8 & $(4.6)$ & 26 & $(15.0)$ & $<0.001$ & 1 & - & - \\
\hline
\end{tabular}

(Continued) 
Table I (Continued).

\begin{tabular}{|c|c|c|c|c|c|c|c|c|c|c|}
\hline \multirow[t]{2}{*}{ Variable } & \multicolumn{2}{|c|}{$\begin{array}{c}\text { Total } \\
n=346\end{array}$} & \multicolumn{2}{|c|}{$\begin{array}{c}\text { Cases } \\
\text { Functional } \\
n=173\end{array}$} & \multicolumn{2}{|c|}{$\begin{array}{c}\text { Controls Non } \\
\text { Functional } \\
n=173\end{array}$} & \multirow[t]{2}{*}{$p$ - value } & \multirow[t]{2}{*}{ ORc } & \multirow[t]{2}{*}{$95 \% \mathrm{Cl}$} & \multirow[t]{2}{*}{ p - value } \\
\hline & $\mathbf{n}$ & (\%) & $\mathbf{n}$ & (\%) & $\mathbf{n}$ & (\%) & & & & \\
\hline \multicolumn{11}{|l|}{ Chronic kidney disease } \\
\hline Absent & 332 & $(96.0)$ & 168 & $(97.1)$ & 164 & $(94.8)$ & 0.75 & 1.84 & $0.60-5.619$ & 0.28 \\
\hline Present & 14 & $(4.0)$ & 5 & $(2.9)$ & 9 & $(5.2)$ & 0.13 & 1 & - & - \\
\hline \multicolumn{11}{|l|}{ Chronic respiratory disease } \\
\hline Absent & 312 & $(90.2)$ & 158 & (91.3) & 154 & $(89.0)$ & 0.74 & 1.30 & $0.63-2.65$ & 0.47 \\
\hline Present & 34 & $(9.8)$ & 15 & $(8.7)$ & 19 & $(I I .0)$ & 0.33 & 1 & - & - \\
\hline \multicolumn{11}{|l|}{ General morbidity } \\
\hline Low:<3 points & 289 & $(83.5)$ & 154 & $(89.0)$ & 135 & $(78.0)$ & 0.11 & 2.28 & $1.25-4.14$ & 0.007 \\
\hline High: $>3$ points & 57 & $(16.5)$ & 19 & $(11.0)$ & 38 & $(22.0)$ & $<0.001$ & 1 & - & - \\
\hline \multicolumn{11}{|l|}{ Functionality prior to the hip fracture } \\
\hline Functional (>90 points) & 235 & (67.9) & 140 & $(80.9)$ & 95 & (54.9) & $<0.001$ & 3.48 & $2.14-5.64$ & $<0.001$ \\
\hline Non Functional ( $<90$ points) & 111 & $(32.1)$ & 33 & $(19.1)$ & 78 & $(45.1)$ & $<0.001$ & 1 & - & - \\
\hline \multicolumn{11}{|l|}{ Subcapital fracture } \\
\hline Present & 34 & $(9.8)$ & 17 & $(9.8)$ & 17 & $(9.8)$ & 1.0 & 1.00 & $0.49-2.03$ & 1.0 \\
\hline Absent & 312 & $(90.2)$ & 156 & $(90.2)$ & 156 & $(90.2)$ & 1.0 & 1 & - & - \\
\hline \multicolumn{11}{|l|}{ Transcervical fracture } \\
\hline Present & 56 & $(16.2)$ & 34 & $(19.7)$ & 22 & $(12.7)$ & 0.023 & 1.67 & $0.93-3.01$ & 0.08 \\
\hline Absent & 290 & $(83.8)$ & 139 & $(80.3)$ & 151 & $(87.3)$ & 0.31 & 1 & - & - \\
\hline \multicolumn{11}{|l|}{ Basicervical fracture } \\
\hline Present & 29 & $(8.4)$ & 16 & $(9.2)$ & 13 & $(7.5)$ & 0.43 & 1.25 & $0.58-2.69$ & 0.56 \\
\hline Absent & 317 & (91.6) & 157 & $(90.8)$ & 160 & $(92.5)$ & 0.81 & 1 & - & - \\
\hline \multicolumn{11}{|l|}{ Transtrochanteric fracture } \\
\hline Present & 207 & $(59.8)$ & 94 & $(54.3)$ & 113 & $(65.3)$ & 0.06 & 0.63 & $0.4 I-0.97$ & 0.038 \\
\hline Absent & 139 & $(40.2)$ & 79 & $(45.7)$ & 60 & $(34.7)$ & 0.023 & 1 & - & - \\
\hline \multicolumn{11}{|l|}{ Subtrochanteric fracture } \\
\hline Present & 20 & $(5.8)$ & 12 & $(6.9)$ & 8 & $(4.6)$ & 0.20 & 1.53 & $0.61-3.86$ & 0.36 \\
\hline Absent & 326 & $(94.2)$ & 161 & $(93.1)$ & 165 & $(95.4)$ & 0.75 & 1 & - & - \\
\hline \multicolumn{11}{|l|}{ Nutritional status } \\
\hline Normal (>24 points) & 217 & $(62.7)$ & 150 & $(86.7)$ & 67 & $(38.7)$ & $<0.001$ & 10.31 & $6.04-|7.6|$ & $<0.001$ \\
\hline Nutritional risk/Malnutrition ( $\leq 24$ points) & 129 & $(37.3)$ & 23 & $(13.3)$ & 106 & $(61.3)$ & $<0.001$ & 1 & - & - \\
\hline \multicolumn{11}{|l|}{ Hip pain } \\
\hline No pain (0 points) & 154 & $(44.4)$ & 88 & $(50.9)$ & 66 & $(38.2)$ & 0.012 & 3.75 & $1.76-8.02$ & 0.001 \\
\hline $\operatorname{Low}(I-3$ points $)$ & 80 & $(23.1)$ & 44 & $(25.4)$ & 36 & $(20.8)$ & 0.20 & 3.44 & $1.52-7.79$ & 0.003 \\
\hline Moderate (4-7 points) & 70 & $(20.2)$ & 30 & $(17.3)$ & 40 & $(23.1)$ & 0.09 & 2.11 & $0.91-4.87$ & 0.07 \\
\hline High (8-10 points) & 42 & $(9.2)$ & 11 & (6.4) & 31 & $(17.9)$ & $<0.001$ & 1 & - & - \\
\hline \multicolumn{11}{|l|}{ Rehabilitation time } \\
\hline$\geq$ I month & 57 & $(16.5)$ & 39 & $(22.5)$ & 18 & $(10.4)$ & $<0.001$ & 2.50 & $1.36-4.58$ & 0.003 \\
\hline$<$ I month & 289 & $(83.5)$ & 134 & $(77.5)$ & 155 & (89.6) & 0.08 & 1 & - & - \\
\hline
\end{tabular}

(Continued) 
Table I (Continued).

\begin{tabular}{|c|c|c|c|c|c|c|c|c|c|c|}
\hline \multirow[t]{2}{*}{ Variable } & \multicolumn{2}{|c|}{$\begin{array}{c}\text { Total } \\
n=346\end{array}$} & \multicolumn{2}{|c|}{$\begin{array}{c}\text { Cases } \\
\text { Functional } \\
n=173\end{array}$} & \multicolumn{2}{|c|}{$\begin{array}{c}\text { Controls Non } \\
\text { Functional } \\
n=173\end{array}$} & \multirow[t]{2}{*}{$p$ - value } & \multirow[t]{2}{*}{ ORc } & \multirow[t]{2}{*}{$95 \% \mathrm{Cl}$} & \multirow[t]{2}{*}{ p - value } \\
\hline & $\mathbf{n}$ & (\%) & $\mathbf{n}$ & (\%) & $\mathbf{n}$ & (\%) & & & & \\
\hline \multicolumn{11}{|l|}{ Social support } \\
\hline High ( $\geq 57$ points) & 118 & $(34.1)$ & 68 & $(39.3)$ & 50 & $(28.9)$ & 0.019 & 2.61 & $1.53-4.45$ & $<0.001$ \\
\hline Moderate (44-56 points) & 114 & (32.9) & 66 & $(38.2)$ & 48 & $(27.7)$ & 0.017 & 2.64 & $1.54-4.52$ & $<0.001$ \\
\hline Low ( $\leq 43$ points $)$ & 114 & (32.9) & 39 & $(22.5)$ & 75 & $(43.4)$ & $<0.001$ & I & - & - \\
\hline \multicolumn{11}{|l|}{ Loneliness } \\
\hline Never (<20 points) & 236 & $(68.2)$ & $|3|$ & $(75.7)$ & 105 & $(60.7)$ & 0.017 & 2.91 & $0.73-11.53$ & 0.12 \\
\hline Rarely (20-39 points) & 69 & $(19.9)$ & 28 & $(16.2)$ & $4 I$ & $(23.7)$ & 0.027 & 1.59 & $0.37-6.69$ & 0.52 \\
\hline Sometimes (40-59 points) & 31 & $(9.0)$ & 11 & $(6.4)$ & 20 & $(I I .6)$ & 0.022 & 1.28 & $0.27-5.98$ & 0.75 \\
\hline Most of the time ( $\geq 60$ points) & 10 & (2.9) & 3 & $(1.7)$ & 7 & $(4.0)$ & 0.07 & I & - & - \\
\hline \multicolumn{11}{|l|}{ Current depressive symptoms } \\
\hline Absent & 191 & $(55.2)$ & 129 & $(74.6)$ & 62 & $(35.8)$ & $<0.001$ & 5.24 & $3.30-8.33$ & $<0.001$ \\
\hline Present & 155 & $(44.8)$ & 44 & $(25.4)$ & 111 & $(64.2)$ & $<0.001$ & I & - & - \\
\hline \multicolumn{11}{|l|}{ Cognitive function } \\
\hline No cognitive impairment & 232 & $(67.1)$ & 134 & $(77.5)$ & 98 & $(56.6)$ & 0.001 & 2.63 & $1.64-4.19$ & $<0.001$ \\
\hline Cognitive impairment & 114 & (32.9) & 39 & $(22.5)$ & 75 & $(43.4)$ & $<0.001$ & 1 & - & - \\
\hline \multicolumn{11}{|l|}{ Self-efficacy for activities of daily living } \\
\hline High ( $\geq 57$ points) & 221 & $(63.9)$ & 150 & $(86.7)$ & 71 & $(41.0)$ & $<0.001$ & 9.36 & $5.49-15.97$ & $<0.001$ \\
\hline Moderate and Low (<57 points) & 125 & $(36.1)$ & 23 & $(13.3)$ & 102 & $(59.0)$ & $<0.001$ & I & - & - \\
\hline \multicolumn{11}{|l|}{ Self-perception of health } \\
\hline Very good & 49 & $(14.2)$ & 33 & $(19.1)$ & 16 & $(9.2)$ & 0.001 & 7.42 & $3.41-16.13$ & $<0.001$ \\
\hline Good & 108 & $(31.2)$ & 75 & $(43.4)$ & 33 & $(19.1)$ & $<0.001$ & 8.18 & $4.30-15.55$ & $<0.001$ \\
\hline Poor & 97 & $(28.0)$ & 45 & $(26.0)$ & 52 & $(30.1)$ & 0.31 & 3.11 & $1.64-5.88$ & $<0.001$ \\
\hline Very poor & 92 & $(26.6)$ & 20 & $(11.6)$ & 72 & $(4 I .6)$ & $<0.001$ & I & - & - \\
\hline \multicolumn{11}{|l|}{ Fear of falling } \\
\hline Low ( $\leq 44$ points) & 114 & (32.9) & 77 & $(44.5)$ & 37 & $(2 \mathrm{I} .4)$ & $<0.001$ & 4.33 & $2.48-7.57$ & $<0.001$ \\
\hline Moderate (45-55 points) & 121 & $(35.0)$ & 60 & (34.7) & 61 & (35.3) & 0.89 & 2.04 & $1.20-3.49$ & 0.008 \\
\hline High ( $\geq 56$ points $)$ & 111 & $(32.1)$ & 36 & $(20.8)$ & 75 & $(43.4)$ & $<0.001$ & I & - & - \\
\hline
\end{tabular}

Abbreviations: ORc, crude odds ratio; $\mathrm{Cl}$, confidence interval.

functionality prior to the hip fracture, high socioeconomic status, and transcervical fracture are associated with recovered functionality in Mexican older adults after a hip fracture.

Several studies have pointed out the importance of nutritional status for the health of older adults with a hip fracture. In general, the prevalence of malnutrition is high and negatively influences functional recovery and is associated with higher mortality. ${ }^{7}$ People with malnutrition have more complications, such as the anorexia of aging syndrome derived from alterations in sensory organs, edentulism, lack of a main caregiver, and even adverse effects to drugs. ${ }^{7,25}$ Malnutrition involves a caloric deficit that increases catabolism, the presence of sarcopenia, and consequently results in reduced functionality. ${ }^{26}$ On the other hand, adequate nutritional management generates positive effects for functionality. ${ }^{7,27}$ In our study, we found a higher prevalence of malnutrition in nonfunctional people. This condition was the most associated with functionality; therefore, we emphasize its clinical importance since it is potentially modifiable. ${ }^{27}$

We also observed that $9.8 \%$ of the entire sample had some heart disease (myocardial infarction 3.4\%, heart failure $4.3 \%$, arrhythmia $2 \%$ ). The frequency of cardiovascular diseases that we found was not as high as that reported by other authors. ${ }^{28,29}$ It is likely that this is due to the fact 
Table 2 Multivariate Logistic Regression Model of the Strength of Association (Adjusted OR, 95\% Cl) with Functionality After Hip Fracture

\begin{tabular}{|c|c|c|c|c|c|c|}
\hline Variable & $\boldsymbol{\beta}$ & Standard Error & Wald & OR & $95 \% \mathrm{Cl}$ & $p$ - value \\
\hline Constant & -5.37 & 0.71 & 55.82 & 0.005 & - & $<0.001$ \\
\hline $\begin{array}{l}\text { Nutritional status } \\
\text { Normal ( }>24 \text { points) } \\
\text { Nutritional risk/Malnutrition ( } \leq 24 \text { points) }\end{array}$ & 1.573 & 0.326 & 23.312 & $\begin{array}{l}4.81 \\
\text { Ref. }\end{array}$ & $2.54-9.12$ & $<0.001$ \\
\hline $\begin{array}{l}\text { Heart disease } \\
\text { Absent } \\
\text { Present }\end{array}$ & 1.407 & 0.515 & 7.465 & $\begin{array}{l}4.08 \\
\text { Ref. }\end{array}$ & $1.48-11.20$ & 0.006 \\
\hline $\begin{array}{l}\text { Self-efficacy for activities of daily living } \\
\text { High ( } \geq 57 \text { points) } \\
\text { Moderate and Low ( }<57 \text { points) }\end{array}$ & 1.405 & 0.326 & 18.553 & $\begin{array}{l}4.07 \\
\text { Ref. }\end{array}$ & $2.15-7.72$ & $<0.001$ \\
\hline $\begin{array}{l}\text { Current depressive symptoms } \\
\text { Absent } \\
\text { Present }\end{array}$ & 1.097 & 0.290 & 14.302 & $\begin{array}{l}2.99 \\
\text { Ref. }\end{array}$ & $1.69-5.28$ & $<0.001$ \\
\hline $\begin{array}{l}\text { Functionality prior to the hip fracture } \\
\text { Functional ( }>90 \text { points) } \\
\text { Non Functional ( } \leq 90 \text { points) }\end{array}$ & 1.042 & 0.321 & 10.555 & $\begin{array}{l}2.83 \\
\text { Ref. }\end{array}$ & $|.5|-5.3 \mid$ & 0.001 \\
\hline $\begin{array}{l}\text { Socioeconomic level } \\
\text { High } \\
\text { Moderate and low }\end{array}$ & 0.880 & 0.336 & 6.870 & $\begin{array}{l}2.4 \mathrm{I} \\
\text { Ref. }\end{array}$ & $1.24-4.65$ & 0.009 \\
\hline $\begin{array}{l}\text { Transcervical } \\
\text { Present } \\
\text { Absent }\end{array}$ & 0.852 & 0.409 & 4.329 & $\begin{array}{l}2.34 \\
\text { Ref. }\end{array}$ & $1.05-5.22$ & 0.037 \\
\hline
\end{tabular}

Abbreviations: $\mathrm{OR}$, odds ratio; $\mathrm{Cl}$, confidence interval.

that our sample only includes participants whose hip fracture occurred after 3 or more months prior to the study and patients who died from unfavorable health conditions, as well as the patients with dementia, were excluded. Therefore, they did not contribute to the frequency of cardiovascular diseases that we report here. This is supported by the evidence derived from epidemiological studies that have previously linked cardiovascular diseases with dementia ${ }^{30}$ and with high mortality 30 days after hip fracture. ${ }^{29}$ On the other hand, we observed that the specific absence of heart disease contributed to a better functional state after the fracture. This is similar to what was found in other studies where heart disease influenced the functional prognosis and mortality of the patient. ${ }^{31,32}$

Despite the fact that we carried out exhaustive data collection on other comorbidities that have been favorably or unfavorably associated, such as chronic respiratory diseases, ${ }^{33}$ chronic kidney failure, ${ }^{34}$ cerebrovascular disease, cognitive function ${ }^{8}$ and even by number of comorbidities, ${ }^{4,8}$ these were discarded in our final model. We speculate that our findings are not consistent with previous reports due to the time of evolution of the fracture, severity, and low frequency of these comorbidities.

Psychological characteristics such as high level of selfefficacy for activities of daily living and the absence of depression explained favorable changes in functionality. Although it has been pointed out that psychological factors such as fear of falling, depression and perceived health play a role in functional recovery, the existing information that supports this argument is limited. ${ }^{11,35,36}$ Previous evidence indicates that health self-care behaviors are involved in disease and recovery processes. ${ }^{37,38}$ For example, self-efficacy functions as a proximal and direct predictor of intention to carry out any action; it facilitates positive changes in healthrelated behaviors through self-control, as well as changes in effort, time and resilience when facing adversity; ${ }^{39}$ it has even been related to health outcomes and healthy lifestyles. ${ }^{40,41}$ From the search carried out, only one study has documented a significant association between high selfefficacy and a higher probability of recovering locomotion in 
patients who received rehabilitation therapy after a hip fracture; this association persisted even when controlling for functional level prior to the fracture and depressive symptoms. ${ }^{42}$ The results of our study support the influence of self-efficacy in the functional recovery process, demonstrating its association independently of other relevant clinical variables, such as comorbidities and physical symptoms like pain. We also observed that the absence of current depressive symptoms was associated with a better functional status. This concurs with a study by Mossey et al which found that people with few depressive symptoms were three times more likely to walk independently and nine times more likely to return to their pre-fracture level of function. ${ }^{43}$ This association has been confirmed in other studies. ${ }^{11}$ The association we found indicates that people without depressive symptoms are almost twice as likely to have better functionality; therefore, we suggest prioritizing psychological wellbeing to obtain better functional results using intervention strategies focused on treating depression or increasing self-efficacy. ${ }^{44}$

Our final model also indicates that being functional before the hip fracture implies a greater possibility of being functional after the traumatic event, similar to what has been previously reported. ${ }^{9,45}$ It has even been observed that previous functionality is more predictive than cognitive status. ${ }^{45}$ In our study, we excluded patients with dementia to avoid confusion or information biases; however, mild cognitive impairment was measured but was not significant in the final model.

On the other hand, few studies indicate that a higher level of social and socioeconomic support has a positive effect on functional recovery. ${ }^{6,10}$ We only found a significant association with socioeconomic level, which is consistent with a study on predictive factors of pain and functionality that observed an association between low income and functional deterioration $(\mathrm{OR}=1.75 ; 95 \% \mathrm{CI}$ $=1.11-2.76) .{ }^{6}$ Generally, socioeconomic level is measured with economic income; we evaluated this construct through a validated instrument, which represents material and social capital that facilitates access to a set of resources and lifestyles that benefit health. ${ }^{16}$ A probable mechanism behind socioeconomic status as a social factor that favors functional recovery is its positive effect on individuals' psychological state as it can results in psychosocial, quality of life or life-enhancing benefits. ${ }^{46}$

The role of the type of fracture in functionality has been studied less, although this variable has previously been suggested as a predictor. ${ }^{47} \mathrm{We}$ found results similar to other studies that have shown that cervical fractures preserve greater functionality than trochanteric fractures when compared a few months after the fracture. ${ }^{5}$ Older patients more frequently present trochanteric fractures ${ }^{5}$ and more vulnerable health states such as frailty and increased comorbidities. ${ }^{48}$ It is possible that the combined effect of these conditions together with other intermediate variables such as surgical technique, complications and perioperative clinical evolution, are indirectly reflected in the effect of the type of fracture.

We acknowledge limitations in our study. First, the information is limited to the duration of the rehabilitation and we do not have details about this therapy or the immediate multidisciplinary care that the patients received after their hospitalization. Second, the cohort participants who died less than 3 months after their fracture had passed were not eligible for this study. It is possible that the characteristics and exposures of these patients differ from those in our sample and therefore our findings are not as useful for the most vulnerable patients. Third, the generalizability of the findings is limited to the inclusion criteria for the sample: IMSS beneficiaries without dementia and who have survived 3 months or more after their fracture. Generalizing our findings to patients who have different characteristics from those in our study would be difficult. However, IMSS is responsible for serving approximately $50 \%$ of the people aged 60 and older in Mexico City. ${ }^{49}$

\section{Conclusion}

In older adults who have suffered a hip fracture, clinical, psychological and demographic factors are associated with recovered functionality. Particularly: Clinical aspects such as normal nutritional status, not having heart disease, being functional prior to the fracture or presenting a transcervical fracture; psychological traits including high self-efficacy in activities of daily life or absence of depression; and demographic characteristics like high socioeconomic level. These factors may contribute to functional recovery and should be considered as a priority in the care of older adults who have suffered hip fractures.

\section{Ethical Approval}

The study adhered to national and international ethical guidelines and regulations in human research and was established according to the ethical guidelines of the Helsinki Declaration. All participants signed an informed consent form before participating. The study was approved by the IMSS National Committee for Scientific Research (Comité Nacional de Investigación Científica y Comité de Ética en Investigación 
en Salud COMBIOETICA09CE101520130424), registration number 2016-785-036.

\section{Acknowledgments}

The publication of this paper was supported by a grant from the Secretaria de Educación, Ciencia, Tecnología e Innovación de la Ciudad de México CM-SECTEI/200/ 2020 "Red Colaborativa de Investigación Traslacional para el Envejecimiento Saludable de la Ciudad de México (RECITES)".

\section{Funding}

This study was financed with grants from the Fund for the Development of Technological Research and Development Protocols on Priority Health Issues, IMSS (FIS/IMSS/ PROT/PRIO/16/060). The findings and conclusions in this article are those of the authors and do not necessarily represent the views of funding agencies.

\section{Disclosure}

The authors report no conflicts of interest in this work.

\section{References}

1. Cauley JA. Burden of hip fracture on disability. Lancet Public Health. 2017;2(5):e209-e210. doi:10.1016/S2468-2667(17)30067-1

2. van Balen R, Steyerberg EW, Polder JJ, Ribbers TL, Habbema JD, Cools HJ. Hip fracture in elderly patients: outcomes for function, quality of life, and type of residence. Clin Orthop Relat Res. 2001;390:232-243.

3. Dyer SM, Crotty M, Fairhall N, et al. A critical review of the long-term disability outcomes following hip fracture. BMC Geriatr. 2016;16(1):158. doi:10.1186/s12877-016-0332-0

4. Schulz C, Büchele G, Rehm M, et al. Patient characteristics as indicator for care dependence after hip fracture: a Retrospective Cohort Study using health insurance claims data from Germany. $J$ Am Med Dir Assoc. 2019;20(4):451-455.e3. doi:10.1016/j.jamda.2018.09.029

5. Takahashi A, Naruse H, Kitade I, et al. Functional outcomes after the treatment of hip fracture. PLoS One. 2020;15(7):e0236652. doi:10.1371/journal.pone. 0236652

6. Orive M, Anton-Ladislao A, García-Gutiérrez S, et al. Prospective study of predictive factors of changes in pain and hip function after hip fracture among the elderly. Osteoporos Int. 2016;27(2):527-536. doi:10.1007/s00198-015-3267-y

7. Malafarina V, Reginster J-Y, Cabrerizo S, et al. Nutritional status and nutritional treatment are related to outcomes and mortality in older adults with hip fracture. Nutrients. 2018;10:5. doi:10.3390/ nu10050555

8. Tarazona-Santabalbina FJ, Belenguer-Varea Á, Rovira-Daudi E, et al. Early interdisciplinary hospital intervention for elderly patients with hip fractures - functional outcome and mortality. Clinics (Sao Paulo). 2012;67(6):547-555. doi:10.6061/clinics/2012(06)02

9. de Miguel Artal M, Roca Chacón O, Martínez-Alonso M, Serrano Godoy M, Mas Atance J, García Gutiérrez R. Hip fracture in the elderly patient: prognostic factors for mortality and functional recovery at one year. Rev Esp Geriatr Gerontol. 2018;53(5):247-254. doi:10.1016/j.regg.2018.04.447
10. Auais M, Al-Zoubi F, Matheson A, Brown K, Magaziner J, French SD. Understanding the role of social factors in recovery after hip fractures: a structured scoping review. Health Soc Care Community. 2019;27(6):1375-1387. doi:10.1111/hsc.12830

11. Givens JL, Sanft TB, Marcantonio ER. Functional recovery after hip fracture: the combined effects of depressive symptoms, cognitive impairment, and delirium. $J$ Am Geriatr Soc. 2008;56 (6): 1075-1079. doi:10.1111/j.1532-5415.2008.01711.x

12. Lwanga SK, Lemeshow S. Sample size determination in health studies: a practical manual/SK Lwanga and S. Lemeshow; 1991. Available from: http://apps.who.int/iris/handle/10665/40062. Accessed March 31, 2013.

13. Infante-Castro CI, Rojano-Mejía D, Ayala-Vázquez G, AguilarEsparza G. [Functional prognostic factors in older adults with hip fracture]. Cirugia Y Cirujanos. 2013;81(2):125-130. Spanish.

14. Shah S, Vanclay F, Cooper B. Improving the sensitivity of the Barthel index for stroke rehabilitation. J Clin Epidemiol. 1989;42 (8):703-709.

15. Uyttenboogaart M, Stewart RE, Vroomen PCAJ, De Keyser J, Luijckx G-J. Optimizing cutoff scores for the Barthel index and the modified Rankin scale for defining outcome in acute stroke trials. Stroke. septiembre de. 2005;36(9):1984-1987.

16. AMAI. Cuestionario de Nivel socioeconómico; 2016. Available from: https://www.amai.org/NSE/. Accessed October 20, 2016.

17. Jensen MP, Turner JA, Romano JM, Fisher LD. Comparative reliability and validity of chronic pain intensity measures. Pain. 1999;83 (2):157-162. doi:10.1016/s0304-3959(99)00101-3

18. Del Refugio Acuña-Gurrola M, González-Celis-Rangel AL. Selfefficacy and social support network in older adults. J Behav Health Soc Issues. 2010;2(2):71-81.

19. Montero López Lena M, Rivera Ledesma A. IMSOL-AM: Escala de Soledad en el Adulto Mayor. In: Gonzalez-Celis-Rangel A. Evaluación En Psicogerontología. México: Manual Moderno; 2009:123-131.

20. Sánchez-García S, Juárez-Cedillo T, Gallegos-Carrillo K, Gallo JJ, Wagner FA, García-Peña C. Frecuencia de los síntomas depresivos entre adultos mayores de la Ciudad de México. Salud mental. 2012;35(1):71-77.

21. de Beaman SR, Beaman PE, Garcia-Peña $C$, et al. Validation of a Modified Version of the Mini-Mental State Examination (MMSE) in Spanish. Aging Neuropsychol Cognition. 2004;11(1):1-11. doi:10.1076/anec.11.1.1.29366

22. Gonzalez-Celis-Rangel A. Autoeficacia para realizar actividades cotidianas (AeRAC) en ancianos mexicanos. In: Gonzalez-CelisRangel A. Evaluación En Psicogerontología. México: Manual Moderno; 2009:47-75.

23. Beaman PE, Reyes-Frausto S, García-Peña C. Validation of the health perceptions questionnaire for an older Mexican population. Psychol Rep. 2003;92(3 Pt 1):723-734. doi:10.2466/pr0.2003.92.3.723

24. Yardley L, Beyer N, Hauer K, Kempen G, Piot-Ziegler C, Todd C. Development and initial validation of the Falls Efficacy Scale-International (FES-I). Age Ageing. 2005;34(6):614-619. doi:10.1093/ageing/afi196

25. García Lázaro M, Montero Pérez-Barquero M, Carpintero Benítez P. [The role of malnutrition and other medical factors in the evolution of patients with hip fracture]. An Med Interna. 2004;21(11):557-563. Spanish. doi:10.4321/s0212-71992004001100009

26. Steihaug OM, Gjesdal CG, Bogen B, et al. Does sarcopenia predict change in mobility after hip fracture? A multicenter observational study with one-year follow-up. BMC Geriatr. 2018;18(1):65. doi:10.1186/s12877-018-0755-x

27. Duncan DG, Beck SJ, Hood K, Johansen A. Using dietetic assistants to improve the outcome of hip fracture: a randomised controlled trial of nutritional support in an acute trauma ward. Age Ageing. 2006;35 (2):148-153. doi:10.1093/ageing/afj011 
28. Xu B, Han L, Liu H, et al. Cardiovascular disease and hip fracture among older inpatients in Beijing, China. Biomed Res Int. 2013;2013:493696.

29. Norring-Agerskov D, Madsen CM, Bathum L, et al. History of cardiovascular disease and cardiovascular biomarkers are associated with 30-day mortality in patients with hip fracture. Osteoporos Int. 2019;30(9):1767-1778.

30. Suemoto CK, Ferretti RE, Grinberg LT, et al. Association between cardiovascular disease and dementia. Dement Neuropsychol. 2009;3 (4):308-314.

31. Reguant F, Bosch J, Montesinos J, Arnau A, Ruiz C, Esquius P. [Prognostic factors for mortality in elderly patients with hip fracture]. Rev Esp Anestesiol Reanim. 2012;59(6):289-298. Spanish doi:10.1016/j.redar.2012.03.006

32. Mathew R, Hsu W-H, Young Y. Effect of comorbidity on functional recovery after hip fracture in the elderly. Am J Phys Med Rehabil. 2013;92(8):686-696. doi:10.1097/PHM.0b013e318282bc67

33. Henderson CY, Ryan JP. Predicting mortality following hip fracture: an analysis of comorbidities and complications. Ir J Med Sci. 2015;184(3):667-671. doi:10.1007/s11845-015-1271-z

34. Orabona N, Bove A, Smeraglia F, et al. The Impact of hemodialysis on mortality and personal independence after hip fracture. A Prospective Matched Cohort Study. J Orthop Trauma. 2019;33 (11):577-582. doi:10.1097/BOT.0000000000001556

35. Oude Voshaar RC, Banerjee S, Horan M, et al. Fear of falling more important than pain and depression for functional recovery after surgery for hip fracture in older people. Psychol Med. 2006;36 (11):1635-1645. doi:10.1017/S0033291706008270

36. Proctor R, Wade R, Woodward Y, et al. The impact of psychological factors in recovery following surgery for hip fracture. Disabil Rehabil. 2008;30(9):716-722. doi:10.1080/09638280701403536

37. Machón M, Vergara I, Dorronsoro M, Vrotsou K, Larrañaga I. Selfperceived health in functionally independent older people: associated factors. BMC Geriatr. 2016;16(1):66. doi:10.1186/s12877-016-0239-9

38. Levy BR, Slade MD, Kasl SV. Longitudinal benefit of positive self-perceptions of aging on functional health. J Gerontol B Psychol Sci Soc Sci. 2002;57(5):P409-417. doi:10.1093/geronb/57.5.p409

39. Bandura A. Self-efficacy: toward a unifying theory of behavioral change. Psychol Rev. 1977;84(2):191-215. doi:10.1037//0033$295 \times .84 .2 .191$
40. Anderson ES, Winett RA, Wojcik JR. Self-regulation, self-efficacy, outcome expectations, and social support: social cognitive theory and nutrition behavior. Ann Behav Med. 2007;34(3):304-312. doi: $10.1007 / \mathrm{bf02874555}$

41. Jeng C, Braun LT. Bandura's self-efficacy theory: a guide for cardiac rehabilitation nursing practice. J Holist Nurs. 1994;12(4):425-436. doi:10.1177/089801019401200411

42. Fortinsky RH, Bohannon RW, Litt MD, et al. Rehabilitation therapy self-efficacy and functional recovery after hip fracture. Int $J$ Rehabil Res. 2002;25(3):241-246.

43. Mossey JM, Knott K, Craik R. The effects of persistent depressive symptoms on hip fracture recovery. J Gerontol. 1990;45(5):M163168.

44. Langford D, Edwards N, Gray SM, Fleig L, Ashe MC. "Life goes on." everyday tasks, coping self-efficacy, and independence: exploring older adults' recovery from hip fracture. Qual Health Res. 2018;28(8):1255-1266. doi:10.1177/1049732318755675

45. Beloosesky Y, Grinblat J, Epelboym B, Weiss A, Grosman B, Hendel D. Functional gain of hip fracture patients in different cognitive and functional groups. Clin Rehabil. 2002;16(3):321-328. doi:10.1191/0269215502cr497oa

46. Allegrante JP, Peterson MGE, Cornell CN, et al. Methodological challenges of multiple-component intervention: lessons learned from a randomized controlled trial of functional recovery after hip fracture. HSS J. 2007;3(1):63-70. doi:10.1007/s11420-006-9036-x

47. Koval KJ, Skovron ML, Aharonoff GB, Zuckerman JD. Predictors of funcional recovery after hip fracture in the elderly. Clin Orthop Relat Res. 1998;348:22-28.

48. Sánchez-García S, Sánchez-Arenas R, García-Peña C, et al. Frailty among community-dwelling elderly Mexican people: prevalence and association with sociodemographic characteristics, health state and the use of health services. Geriatr Gerontol Int. 2014;14(2):395-402. doi:10.1111/ggi.12114

49. Instituto Nacional de Salud Pública. Encuesta Nacional de Salud y Nutrición. Resultados Por Entidad Federativa, Distrito Federal. Instituto Nacional de Salud Pública. 2012:2013.
Clinical Interventions in Aging

\section{Publish your work in this journal}

Clinical Interventions in Aging is an international, peer-reviewed journal focusing on evidence-based reports on the value or lack thereof of treatments intended to prevent or delay the onset of maladaptive correlates of aging in human beings. This journal is indexed on PubMed Central, MedLine, CAS, Scopus and the Elsevier
Bibliographic databases. The manuscript management system is completely online and includes a very quick and fair peer-review system, which is all easy to use. Visit http://www.dovepress.com/ testimonials.php to read real quotes from published authors. 